\title{
Modeling and Analysis of Rumor Spreading with Social Reinforcement Mechanism
}

\author{
Yuan Xu, ${ }^{1,2}$ Renjie Mei $\left(D,{ }^{3}\right.$ Yujie Yang, ${ }^{3}$ and Zhengmin Kong $\mathbb{D}^{3}$ \\ ${ }^{1}$ College of Literature and Journalism, Minzu University of China, Beijing 100081, China \\ ${ }^{2}$ Liberal Arts College, Yangtze University, Jingzhou 434023, China \\ ${ }^{3}$ School of Electrical Engineering and Automation, Wuhan University, Wuhan 430072, China \\ Correspondence should be addressed to Zhengmin Kong; zmkong@whu.edu.cn
}

Received 23 June 2019; Accepted 9 August 2019; Published 17 December 2019

Academic Editor: Antonio Scarfone

Copyright (c) 2019 Yuan Xu et al. This is an open access article distributed under the Creative Commons Attribution License, which permits unrestricted use, distribution, and reproduction in any medium, provided the original work is properly cited.

\begin{abstract}
It is of great practical significance to figure out the propagation mechanism and outbreak condition of rumor spreading on online social networks. In our paper, we propose a multi-state reinforcement diffusion model for rumor spreading, in which the reinforcement mechanism is introduced to depict individual willingness towards rumor spreading. Multiple intermediate states are introduced to characterize the process that an individual's diffusion willingness is enhanced step by step. We study the rumor spreading process with the proposed reinforcement diffusion mechanism on two typical networks. The outbreak thresholds of rumor spreading on both two networks are obtained. Numerical simulations and Monte Carlo simulations are conducted to illustrate the spreading process and verify the correctness of theoretical results. We believe that our work will shed some light on understanding how human sociality affects the rumor spreading on online social networks.
\end{abstract}

\section{Introduction}

The rumor spreading is one of the basic mechanisms for information dissemination $[1,2]$. It is of great importance to understand the mechanisms of the spreading dynamics and the factors that affect it, such as media coverage [3, 4]. With the advent of the information era, online social networks such as Facebook, Weibo, and WeChat have become one of the most important mediums for the dissemination of rumors [5]. Due to the complexity of propagation mechanism and the diversity of network structure, it is hard to control the speed and the range of rumor spreading. Meanwhile, understanding the path of rumor spreading and modeling the diffusion process are the important basis to control and suppress the rumor spreading on online social networks.

The study of modeling and analysis of rumor spreading process have attracted much attention in recent years. Doerr et al. [6] proposed that rumor spreading is extremely fast on social networks and validated it with mathematical analysis. Abstracting the propagation problem between related things as the propagation dynamics problem on the network is a common and efficient research method. In [7], the authors abstracted the objective process of rumor propagation and established a mathematical model to understand the influence of various related factors, such as transmission rate and mortality rate. Since there are some similarities between the diffusion mechanism of the rumor and the infectious disease, the paradigmatic models of epidemiology such as SusceptibleInfected-Susceptible (SIS) [8, 9] and Susceptible-InfectedRemoved $(S I R)[10,11]$ are widely employed to the study of rumor spreading.

The above models are well-known as compartment models. The extensions of these models can be found in a vast body of studies $[12,13]$. A delayed SVEIR worm propagation model with saturated incidence was investigated in [12]. In addition, integrable deformations of the Kermack-McKendrick model for epidemics were proposed and analyzed in [13]. Importantly, researchers show great interest in the individual characteristic of rumor spreading and extend the paradiamatic models with more compartments for characterizing rumor spreading process. The Susceptible-Infected-Hibernator-Removed (SIHR) model was established in [14], with the hibernation chamber $H$ contained the characteristics of intermittent sleepiness of individuals who spread rumors. This model demonstrates that 


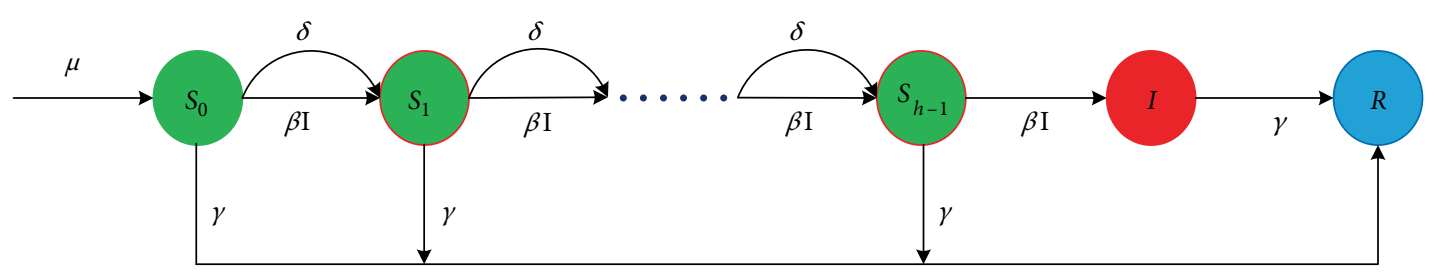

FIGURE 1: The state transition process of individuals involved in rumor spreading.

the spreaders did not continue to spread during the diffusion process. $\mathrm{Hu}$ et al. [15] established a Susceptible-HesitatingAffected-Resistant (SHAR) model to depict individuals' different attitudes toward rumor spreading.

In addition to individual factors, the social factors have been acknowledged as indispensable ingredients of the rumor diffusion process [16-18]. As revealed by many researchers, the social reinforcement effect is one of the key factors in the rumor spreading process on social networks. Wang et al. [17] proposed a rumor propagation model considering the negative and positive double social reinforcement mechanism on the individual propagation behavior. Ma et al. [18] presented a novel model based on the generation function and cavity method developed from statistical physics of disordered system. Their results indicated that decreasing the positive reinforcement factor or increasing the negative reinforcement factor can suppress the rumor spreading effectively. However, it should be pointed out that the above studies did not analyze the reinforcement elements of rumor propagation, nor paid attention to the reinforcement process of rumor spreading. Particularly, the mechanism of social reinforcement and the potential roles of it on the rumor spreading process need to be further explored.

On the other hand, it is necessary to consider the characteristic of social networks for more accurately characterizing the rumor spreading. In most previous studies, the topology structure of propagation network is assumed to be static or quasi-static, while its dynamic nature is always neglected [19]. In practice, the topology of social network is not fixed, and the effective connections between individuals at different periods tend to be time-varying $[20,21]$. Therefore, the topological characteristic of the network is significant for understanding the rumor propagation process. In recent years, a large number of studies on time-varying networks driven by individual activities have emerged [22-24]. In the activity-driven (AD) framework, each individual is endowed with a specific activity rate. At each time step, an individual may become active and generate finite connections with other individuals randomly selected from the network. The corresponding generated network is called as the activity driven network. As the AD model can describe some characteristics of the online social networks, it becomes a hot topic in recent studies. However, the studies that incorporate the time-varying networks with the social reinforcement effect are rare, and thus worth further discussion.

In our paper, we propose a multi-state social reinforcement mechanism for the rumor spreading on both the random network and the AD network. The mechanism focuses on the process in which individuals' willingness to spread a rumor is gradually strengthened by both internal and external factors. This is motivated by the fact that people tend to believe and spread a rumor if they are repeatedly exposed to it. In addition to the external reinforcement, individuals may spontaneously enhance their willingness to spread due to their personal interest in some topics. We attribute individuals with different spreading willingness to different compartments. The individual spreading behavior was triggered by the external reinforcement. In this way, we extend the typical SIR spreading model to the multi-susceptibleinfected-removed ( $m S I R)$ model. Also, we study the rumor propagation dynamics with the enhancement mechanism on the time-varying networks. A dynamic system with a comparable time-scale between the spreading of rumor and the evolving of the network topology is investigated. We derive the outbreak threshold of the rumor spreading on two typical networks, i.e., random networks and $\mathrm{AD}$ networks. The effects of propagation parameters and network parameters on rumor spreading are also discussed. Finally, we perform numerical simulations and Monte Carlo simulations to illustrate and validate theoretical results.

The rest of the paper is organized as follows. In Section 2 , we introduce the social reinforcement mechanism of the rumor spreading. In Section 3, we establish the mathematical model on both random networks and AD networks, and derive the corresponding outbreak thresholds. The numerical experiments and the Monte Carlo simulation analysis are presented in Section 4. Conclusions are drawn in Section 5.

\section{The Compartment Model}

We consider a rumor spreads on a social network of $N$ individuals who interact with their neighbors with equal probability. During the process of rumor spreading, each individual can be one of three states: $S$ (susceptible), $I$ (infected) and $R$ (removed). A susceptible individual in contact with an infectious one becomes infected with a given infection rate. Individuals in $R$ state are not interested in the rumor and no longer pay attention to it. Specifically, we divide $S$ into $S_{i}(i=0,1, \ldots, h-1)$ according to different levels of individual willingness to spread rumor under the reinforcement mechanism. The state transition process of individuals is shown in Figure 1. An individual in the state $S_{k}$ will enter into the state $S_{k+1}$ with probability $\beta$ once it interacts with an infected one. Meanwhile, An $S_{k}$ individual could also be reinforced by themselves and enters into state $S_{k+1}$ with a probability $\delta$. Both susceptible and infectious individuals will lose their interest in rumor and transfer into $R$ with a probability 
$\gamma$. In addition, new individuals enter into the rumor propagation network with the rate $\mu$.

\section{Main Results}

3.1. The Rumor Spreading on a Random Network. Firstly, we consider rumor spreading on a random network with an average degree of $\langle k\rangle$. Since individuals in $R$ are inactive in the rumor spreading process, we only focus on the proportion of susceptible and infected individuals in the propagation process. According to the individual state transition mechanism in Section 2, we can obtain the following dynamic model of rumor spreading on the random network.

$$
\begin{aligned}
\dot{S}_{0}(t) & =\mu-\beta\langle k\rangle S_{0}(t) I(t)-\delta S_{0}(t)-\gamma S_{0}(t), \\
\dot{S}_{1}(t) & =\beta\langle k\rangle S_{0}(t) I(t)+\delta S_{0}(t)-\beta\langle k\rangle S_{1}(t) I(t)-\delta S_{1}(t)-\gamma S_{1}(t), \\
& \vdots \\
\dot{S}_{h-1}(t) & =\beta\langle k\rangle S_{h-2}(t) I(t)+\delta S_{h-2}(t)-\beta\langle k\rangle S_{h-1}(t) I(t)-\gamma S_{h-1}(t), \\
\dot{I}(t) & =\beta\langle k\rangle S_{h-1}(t) I(t)-\gamma I(t),
\end{aligned}
$$

where $\mu, \gamma, \beta$ and $\delta$ are respectively birth rate, death rate, infection rate and self-enhancement rate. $S_{i}(t), I(t)$ denote the proportion of individuals in state $S_{i}, I$, respectively. In Eq. (1), the second term on the right-hand side of the first equation describes the state transition from $S_{0}$ to $S_{1}$ due to their connections with $I$ individuals. The third term on the right-hand side of the first equation describes the state transition from $S_{0}$ to $S_{1}$ due to self-enhancement.

Let $X(t)=\left[S_{0}(t), S_{1}(t), \ldots, S_{h-1}(t), I(t)\right]^{T}$, we have $\dot{X}(\infty)=0$ when $t \rightarrow \infty$. Thus the system (1) goes into the steady state. Let $S_{i}=S_{i}(\infty), I=I(\infty), i=0,1, \ldots, h-1$, the relationship between the steady states of the system can be obtained as

$$
\begin{aligned}
S_{0} & =\frac{\mu}{\beta\langle k\rangle I+\gamma+\delta}, \\
S_{i} & =\frac{\beta\langle k\rangle I+\delta}{\beta\langle k\rangle I+\gamma+\delta} S_{i-1}, \quad i=1,2, \ldots, h-2, \\
S_{h-1} & =\frac{\beta\langle k\rangle I+\delta}{\beta\langle k\rangle I+\gamma} S_{h-2} .
\end{aligned}
$$

Obviously, there is a disease-free equilibrium of the system

$$
\begin{aligned}
E^{0} & =\left(S_{0}, S_{1}, \ldots, S_{h-1}, I\right) \\
& =\left[\frac{\mu}{\gamma+\delta}, \frac{\delta \mu}{(\gamma+\delta)^{2}}, \frac{\delta^{2} \mu}{(\gamma+\delta)^{3}}, \ldots, \frac{\delta^{h-2} \mu}{(\gamma+\delta)^{h-1}}, \frac{\mu}{\gamma} \frac{\delta^{h-1}}{(\gamma+\delta)^{h-1}}, 0\right] .
\end{aligned}
$$

The basic reproduction number is an important parameter in rumor spreading analysis. It denotes an average number of susceptible individuals that can be infected by an infected individual in a given time. By exploiting the basic reproduction number, we can obtain the conditions for the outbreak of propagation. The basic reproduction number can be derived by the following method [25].

$$
R_{0}=\rho\left(F V^{-1}\right)
$$

where $F$ is the growth rate of the proportion of individuals in each state when the system is in its equilibrium, $V^{-1}$ is the average length of time that an individual stays in the corresponding state of the system.

Let $x(t)=\left[S_{1}(t), \ldots, S_{h-1}(t), I(t)\right], \mathscr{F}(t)$ denotes the ratio of the individuals entering into the state $x(t)$ at time $t$, and $\mathscr{V}(t)$ is the ratio of the individual leaving the state $x(t)$ at time $t$. According to system (1), we get the following equations.

$$
\begin{gathered}
\mathscr{F}(t)=\left(\begin{array}{l}
\beta\langle k\rangle S_{0}(t) I(t)+\delta S_{0}(t) \\
\vdots \\
\beta\langle k\rangle S_{h-3}(t) I(t)+\delta S_{h-3}(t) \\
\beta\langle k\rangle S_{h-2}(t) I(t)+\delta S_{h-2}(t) \\
\beta\langle k\rangle S_{h-1}(t) I(t)
\end{array}\right), \\
\mathscr{V}(t)=\left(\begin{array}{l}
\beta\langle k\rangle S_{1}(t) I(t)+\delta S_{1}(t)+\gamma S_{1}(t) \\
\vdots \\
\beta\langle k\rangle S_{h-2}(t) I(t)+\delta S_{h-2}(t)+\gamma S_{h-2}(t) \\
\beta\langle k\rangle S_{h-1}(t) I(t)+\gamma S_{h-1}(t) \\
\gamma I(t)
\end{array}\right) .
\end{gathered}
$$

At disease-free equilibrium point $E^{0}$, the Jacobian matrices of $\mathscr{F}(t)$ and $\mathscr{V}(t)$ can be calculated as

$$
\begin{gathered}
F=\left.\frac{\partial \mathscr{F}}{\partial x(t)}\right|_{E^{0}}=\left(\begin{array}{lll}
0 & 0 & \beta\langle k\rangle S_{0} \\
\delta & 0 & \beta\langle k\rangle S_{1} \\
\vdots & \ddots & \vdots \\
0 & 0 & \beta\langle k\rangle S_{h-1}
\end{array}\right), \\
V=\left.\frac{\partial \mathscr{V}}{\partial x(t)}\right|_{E^{0}}=\left(\begin{array}{llll}
\delta+\gamma & & 0 & \beta\langle k\rangle S_{1} \\
\vdots & 0 & \vdots & \vdots \\
0 & \delta+\gamma & 0 & \beta\langle k\rangle S_{h-2} \\
0 & 0 & \gamma & \beta\langle k\rangle S_{h-1} \\
0 & 0 & 0 & \gamma
\end{array}\right) .
\end{gathered}
$$

Based on (4), the basic reproduction number can be calculated as

$$
R_{0}=\rho\left(F V^{-1}\right)=\frac{\beta\langle k\rangle}{\gamma} S_{h-1}=\frac{\beta\langle k\rangle}{\gamma} \frac{\mu}{\gamma} \frac{\delta^{h-1}}{(\delta+\gamma)^{h-1}} .
$$

The rumor spreading will break out on a certain scale if the condition $R_{0}>1$ is satisfied. In this case, we have

$$
\beta>\beta_{c},
$$

where $\beta_{c}=\left(\gamma^{2} /\langle k\rangle \mu\right)\left((\delta+\gamma)^{h-1} / \delta^{h-1}\right)$ is the outbreak threshold.

3.2. The Rumor Spreading on an Activity Driven Network. When the time scale of network evolution is comparable to that of rumor spreading, we have to consider the dynamic process of both rumor propagation and network evolution. In the AD network, an individual becomes active with probability $a$ within a time step $\Delta t$. The active individual will generate $m$ edges randomly connected to $m$ other individuals who can be either active or inactive. All these edges would be deleted at the next time step $t+\Delta t$ and the procedure is iterated. By clustering analysis of the individuals' historical data on the online social network, we can quantify the activity rate of 
all individuals. Without loss of generality, the activity rate of individuals obeys a distribution $P(a)$.

According to the state transition process of individuals in Figure 1, we can establish a discrete dynamic model for the rumor spreading on the AD network.

$$
\begin{aligned}
S_{0, a}^{t+\Delta t}= & S_{0, a}^{t}+\mu_{a} \Delta t-\beta m S_{0, a}^{t} \Delta t a \int I_{a^{\prime}}^{t} d a^{\prime}-\beta m S_{0, a}^{t} \Delta t \int a^{\prime} I_{a^{\prime}}^{t} d a^{\prime} \\
& -\delta S_{0, a}^{t} \Delta t-\gamma S_{0, a}^{t} \Delta t, \\
S_{1, a}^{t+\Delta t}= & S_{1, a}^{t}+\beta m S_{0, a}^{t} \Delta t a \int I_{a^{\prime}}^{t} d a^{\prime}+\beta m S_{0, a}^{t} \Delta t \int a^{\prime} I_{a^{\prime}}^{t} d a^{\prime} \\
& +\delta S_{0, a}^{t} \Delta t-\beta m S_{1, a}^{t} \Delta t a \int I_{a^{\prime}}^{t} d a^{\prime}-\beta m S_{1, a}^{t} \Delta t \int a^{\prime} I_{a^{\prime}}^{t} d a^{\prime} \\
& -\delta S_{1, a}^{t} \Delta t-\gamma S_{1, a}^{t} \Delta t, \\
\vdots & \\
S_{h-1, a}^{t+\Delta t}= & S_{h-1, a}^{t}+\beta m S_{h-2, a}^{t} \Delta t a \int I_{a^{\prime}}^{t} d a^{\prime}+\beta m S_{h-2, a}^{t} \Delta t \int a^{\prime} I_{a^{\prime}}^{t} d a^{\prime} \\
& +\delta S_{h-2, a}^{t} \Delta t-\beta m S_{h-1, a}^{t} \Delta t a \int I_{a^{\prime}}^{t} d a^{\prime}-\beta m S_{h-1, a}^{t} \Delta t \int a^{\prime} I_{a^{\prime}}^{t} d a^{\prime} \\
& -\gamma S_{h-1, a}^{t} \Delta t, \\
I_{a}^{t+\Delta t}= & I_{a}^{t}+\beta m S_{h-1, a}^{t} \Delta t a \int I_{a^{\prime}}^{t} d a^{\prime}+\beta m S_{h-1, a}^{t} \Delta t \int a^{\prime} I_{a^{\prime}}^{t} d a^{\prime}-\gamma \Delta t I_{a}^{t},
\end{aligned}
$$

where $S_{0, a}^{t}$ is the proportion of individuals in $S_{0}$ state with the activity rate $a$ at time $t$. The meaning of $S_{h-1, a}^{t}$ and $I_{a}^{t}$ is similar to that of $S_{0, a}^{t}$. In addition, $\mu_{a}=\mu P_{a}$ is the birth rate of individuals with the activity rate $a$.

The third term on the right side of the first equation in (11) takes into account the probability that an $S_{0}$ individual with activity rate $a$ is active and is enhanced into $S_{1}$ state by connecting to $I$ individuals. The fourth term stems from the fact that a susceptible individual is connected by an active $I$ individual and thus is enhanced.

Theorem 1. The rumor will break out on the AD network if $\beta>\beta_{c}^{A}$, where $\beta_{c}^{A}$ is given by

$$
\beta_{c}^{A}=\frac{\gamma^{2}}{m \mu} \frac{(\delta+\gamma)^{h-1}}{\delta^{h-1}} \frac{1}{\langle a\rangle+\sqrt{\left\langle a^{2}\right\rangle}} .
$$

Proof. According to the Eq. (11), we can obtain the following disease-free equilibrium.

$$
\begin{aligned}
E_{a}^{0} & =\left(S_{0, a}, S_{1, a}, \ldots, S_{h-1, a}, I_{a}\right) \\
& =\left[\frac{\mu P_{a}}{\gamma+\delta}, \frac{\delta \mu P_{a}}{(\gamma+\delta)^{2}}, \frac{\delta^{2} \mu P_{a}}{(\gamma+\delta)^{3}}, \ldots, \frac{\delta^{h-2} \mu P_{a}}{(\gamma+\delta)^{h-1}}, \frac{\mu}{\gamma} \frac{\delta^{h-1} P_{a}}{(\gamma+\delta)^{h-1}}, 0\right] .
\end{aligned}
$$

The infection level $I^{t+\Delta t}$ in the system can be obtained by integrating $I_{a}^{t+\Delta t}$ over all activity spectrum in (11). Also, by defining $\theta^{t}=\int a^{\prime} I_{a^{\prime}}^{t} d a^{\prime}$, the expressions of $I^{t+\Delta t}$ and $\theta^{t}$ can be derived as follows.

$$
\begin{aligned}
I^{t+\Delta t}= & \int I_{a}^{t+\Delta t} d a=I^{t}+\beta m \Delta t\left\langle S_{h-1, a}^{t} a\right\rangle I^{t} \\
& +\beta m S_{h-1}^{t} \Delta t \theta^{t}-\gamma \Delta t I^{t}, \\
\theta^{t+\Delta t}= & \int a^{\prime} I_{a^{\prime}}^{t+\Delta t} d a^{\prime}=\theta^{t}+\beta m \Delta t\left\langle S_{h-1, a}^{t} a^{2}\right\rangle I^{t} \\
& +\beta m \Delta t\left\langle S_{h-1, a}^{t} a\right\rangle \theta^{t}-\gamma \Delta t \theta^{t} .
\end{aligned}
$$

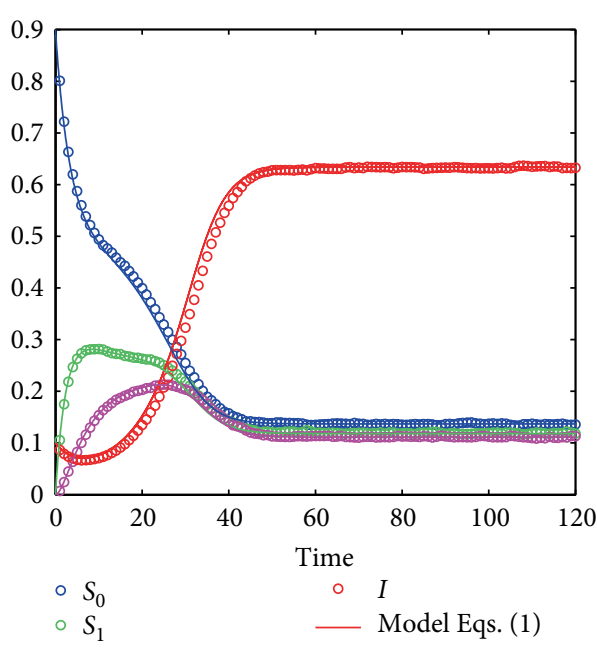

(a)

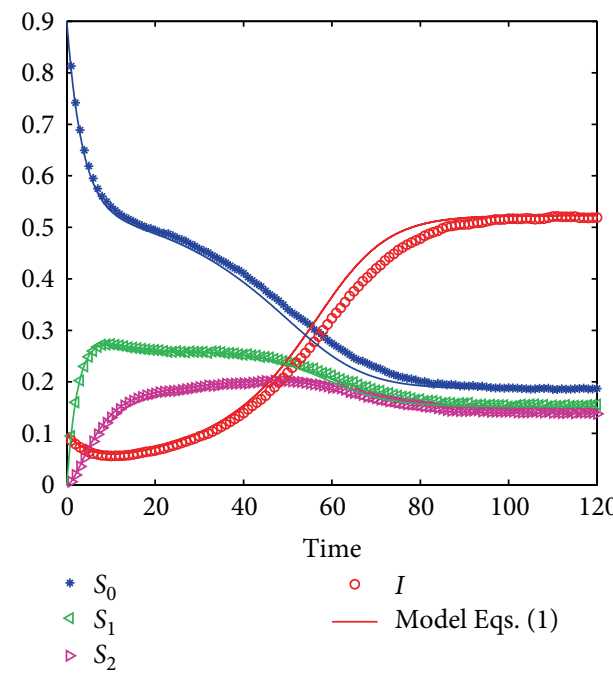

(b)

FIgURE 2: The time evolution of $S_{0}, S_{1}, S_{2}, I$ under two different infection rates on a random network. (a) $\beta=0.15$, (b) $\beta=0.12$. The solid and dotted lines represent numerical simulations and Monte Carlo simulations, respectively. Other parameters are set to be $\delta=0.06, \gamma=\mu=0.1, h=3, N=5000,\langle k\rangle=6, I(0)=0.1$, $S(0)=0.9$.

In the continuous time limit we obtain the following closed system of equations

$$
\begin{aligned}
& \partial_{t} I=\beta m\left\langle S_{h-1, a}^{t} a\right\rangle I+\beta m S_{h-1}^{t} \theta-\gamma I, \\
& \partial_{t} \theta=\beta m\left\langle S_{h-1, a}^{t} a^{2}\right\rangle I+\beta m\left\langle S_{h-1, a}^{t} a\right\rangle \theta-\gamma \theta,
\end{aligned}
$$

whose Jacobian matrix at disease-free equilibrium $E_{a}^{0}$ is derived as

$$
J(I, \theta)=\left.\left[\begin{array}{cc}
\beta m\left\langle S_{h-1, a}^{t} a\right\rangle-\gamma & \beta m S_{h-1}^{t} \\
\beta m\left\langle S_{h-1, a}^{t} a^{2}\right\rangle & \beta m\left\langle S_{h-1, a}^{t} a\right\rangle-\gamma
\end{array}\right]\right|_{S_{h-1, a}^{t}=(\mu / \gamma)\left(\left(\delta^{h-1} P_{a}\right) /(\gamma+\delta)^{h-1}\right)} .
$$

The Jacobian matrix (16) has eigenvalues

$$
\Lambda_{(1,2)}=\beta m \frac{\mu}{\gamma} \frac{\delta^{h-1}}{(\gamma+\delta)^{h-1}}\langle a\rangle-\gamma \pm \beta m \frac{\mu}{\gamma} \frac{\delta^{h-1}}{(\gamma+\delta)^{h-1}} \sqrt{\left\langle a^{2}\right\rangle} .
$$




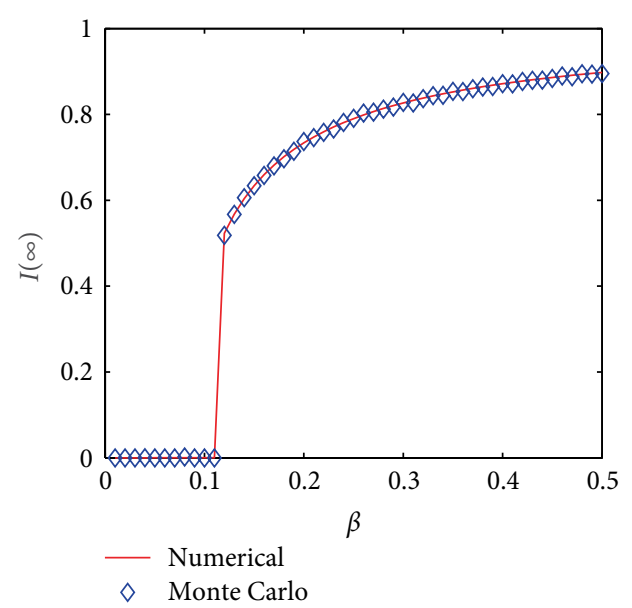

(a)

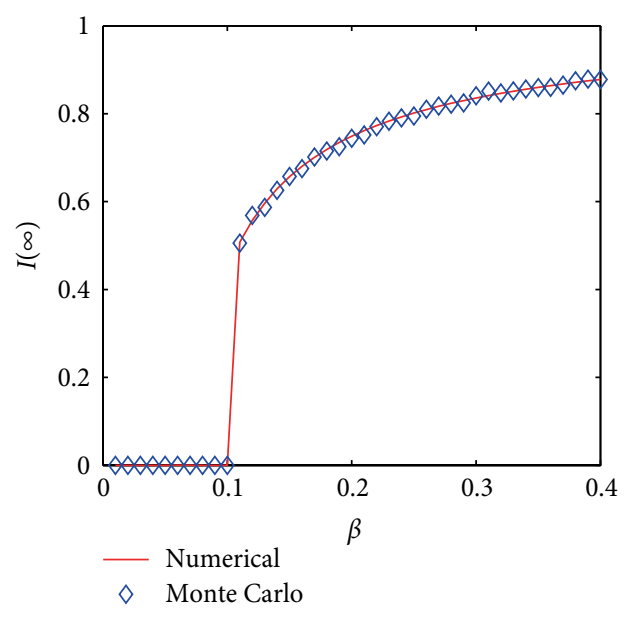

(b)

FIgURE 3: The outbreak scale $I(\infty)$ as a function of the infection rate $\beta$ on the (a) random network with $\langle k\rangle=6$, (b) AD network with $F(a) \propto a^{-2}, a \in\{0.5,0.6,0.7,0.8,0.9\}$. The solid and dotted lines represent numerical simulations and Monte Carlo simulations, respectively. Other parameters are $\delta=0.06$, $\gamma=\mu=0.1, h=3, N=5000, I(0)=0.01, S(0)=0.99$.

The outbreak threshold for the system is obtained requiring the largest eigenvalue to be larger than 0 , which leads to the condition for the presence of an endemic state

$$
\beta>\frac{\gamma^{2}}{m \mu} \frac{(\delta+\gamma)^{h-1}}{\delta^{h-1}} \frac{1}{\langle a\rangle+\sqrt{\left\langle a^{2}\right\rangle}} .
$$

The proof of Theorem 1 is completed.

\section{Simulation and Analysis}

In our simulations, it is assumed that the death rate and birth rate are equal, so that the number of individuals in the system remains constant. The initial infected individuals (spreaders) are chosen randomly in the network. The outbreak thresholds of the random network and the $\mathrm{AD}$ network are $\beta_{c}=(\gamma /\langle k\rangle)\left((\delta+\gamma)^{h-1} / \delta^{h-1}\right)$ and $\beta_{c}^{A}=(\gamma / m)\left((\delta+\gamma)^{h-1} / \delta^{h-1}\right)$ $\left(1 /\left(\langle a\rangle+\sqrt{\left\langle a^{2}\right\rangle}\right)\right)$, respectively.

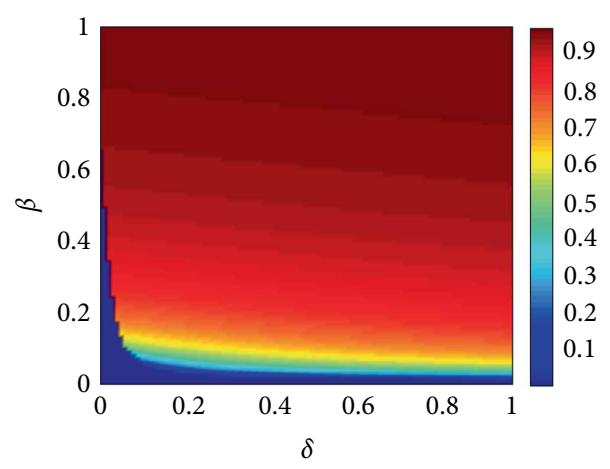

(a)

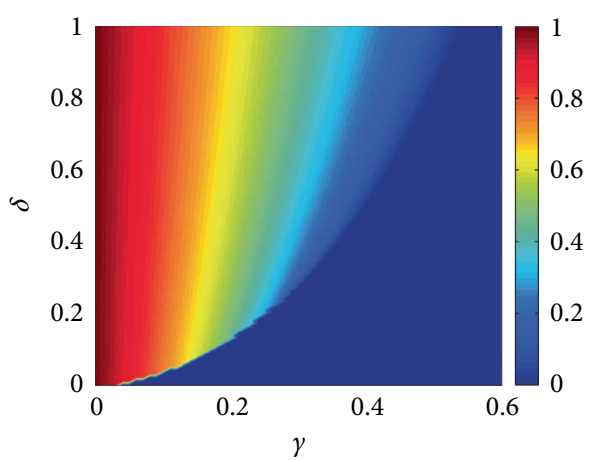

(b)

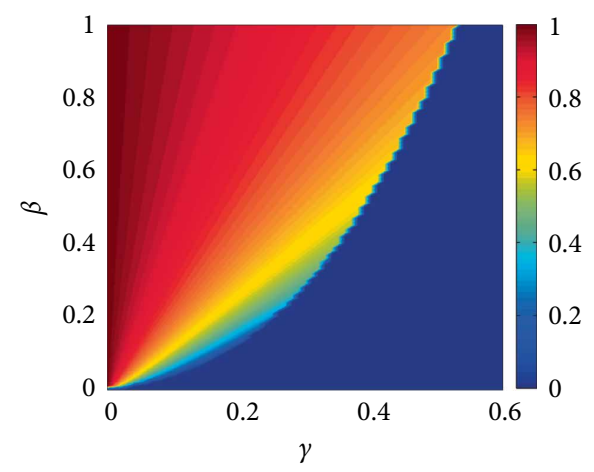

(c)

FIgURE 4: The outbreak scale $I(\infty)$ as a function of propagation parameters $\beta, \delta$, and $\gamma$ on the $\mathrm{AD}$ network with $F(a) \propto a^{-2}$, $a \in\{0.5,0.6,0.7,0.8,0.9\}$. (a) $\gamma=0.1$. (b) $\beta=0.2$. (c) $\delta=0.2$.

4.1. The Performance of Diffusion Parameters. Figure 2 shows the evolution of the proportion of individuals in different states when the rumor spreads on a random network. Two different infection rates are considered and the results of Monte Carlo experiments are averaged over thirty realizations. From Figure 2, it can be seen that the diffusion with a higher infection rate will reach the steady state with a larger outbreak scale in a shorter time. Moreover, a common phenmenon observed in Figures 2(a) and 2(b) is that the proportion of infected individuals $I(t)$ decreases at first and then increases in the initial stage of diffusion. This trend can be understandable in view of the multiple intermediate states of susceptible individuals. Specifically, with $h=3$ in the Figure 2, an $S$ individual needs to go through two intermediate states, $S_{1}$ and $S_{2}$ before it is infected. At the early stage of diffusion, only a small number of susceptible individuals can be infected. Under 


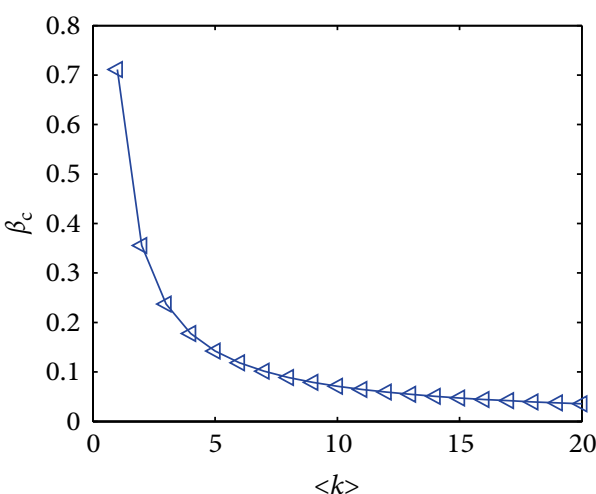

(a)

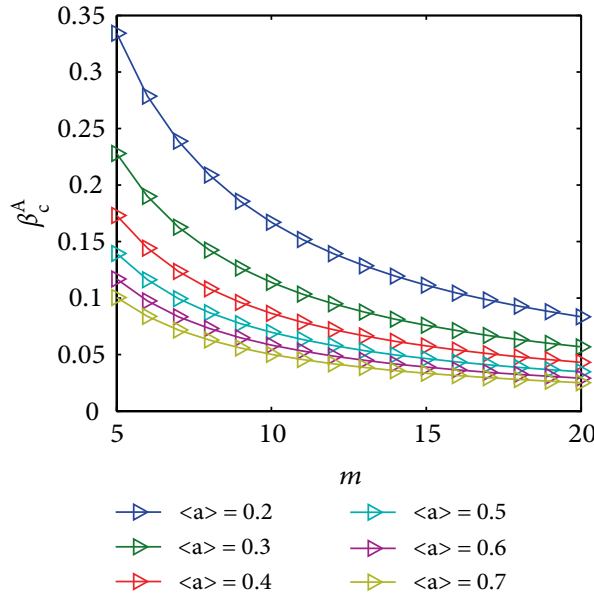

(c)

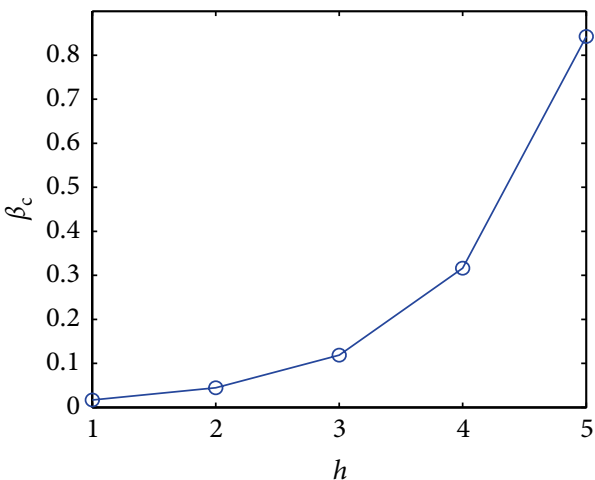

(b)

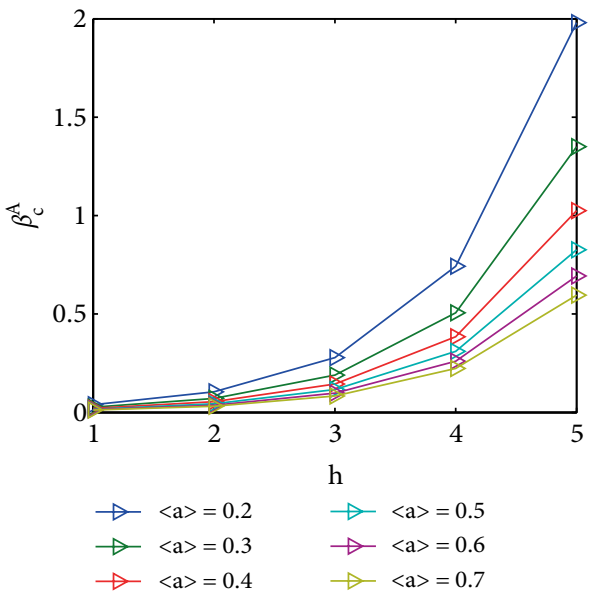

(d)

FIGURE 5: The outbreak thresholds $\beta_{c}$ and $\beta_{c}^{A}$ under different network parameters. (a) The outbreak threshold $\beta_{c}$ against the network average degree $\langle k\rangle, h=3$. (b) The outbreak threshold $\beta_{c}$ against the number of intermediate state $h,\langle k\rangle=6$. Panels (c) and (d) are experiments on AD networks. Other parameters are $\delta=0.06, \gamma=\mu=0.1, N=5000, I(0)=0.01, S(0)=0.99$.

the given simulation parameters, the individuals entering into the state $I$ are less than that removed from the state $I$.

Figure 3 depicts the outbreak scale $I(\infty)$ of rumor spreading as a function of the infection rate $\beta$. When $\beta$ is less than the outbreak threshold $\beta_{c}$, the system reaches the disease-free equilibrium with $I(\infty)=0$. Otherwise, we have $I(\infty)>0$ and the system reaches an endemic equilibrium. In addition, it can be observed that the Monte Carlo results agree well with the theoretical results on both random networks and $\mathrm{AD}$ networks.

Figure 4 presents the outbreak scale $I(\infty)$ as the function of different parameters. From Figure 4(a), it can be seen that the increase of both infection rate $\beta$ and self-enhancement rate $\delta$ can enhance $I(\infty)$. Meanwhile, by comparing Figures $4(\mathrm{~b})$ and $4(\mathrm{c})$, we can see that the impacts of $\beta$ and $\delta$ on $I(\infty)$ are different. As shown in Figure 4(a), for a small $\gamma$, increasing $\delta$ leads to a first-order phase transition of $I(\infty)$. While for a larger $\gamma$ not more than 0.5 , an increase of $\delta$ will bring about a second-order phase transition of $I(\infty)$. However, this phenomenon is different in the Figure 4(b), which indicates a second-order phase transition for small $\beta$ while a first-order transition for a larger $\beta$. In the case of a large $\gamma$, the rumor can hardly break out in the network by improving either the infection rate $\beta$ or the self-enhancement rate $\delta$, which reveals that 'fast food' rumors are hard to be prevalent.
4.2. The Performance of Network Parameters. Figure 5 shows the outbreak thresholds $\beta_{c}$ and $\beta_{c}^{A}$ under different network parameters. In Figure 5(a), we can see that there is a nonlinear and negative correlation between the network average degree $\langle k\rangle$ and the outbreak threshold $\beta_{c}$ of the random network. Specifically, $\beta_{c}$ decreases with the increasing of $\langle k\rangle$ and approaches zero gradually. Meanwhile, the similar relationship exists between the edge number $m$ and the outbreak threshold $\beta_{c}^{A}$ of the AD network in Figure 5(c).

In Figures 5(b) and 5(d), the impacts of the number of intermediate states $h$ on rumor spreading are investigated on both two networks. From Figures 5(b) and 5(d), it is observed that $h$ is nonlinearly and negatively correlated with the outbreak thresholds $\beta_{c}$ and $\beta_{c}^{A}$. Particularly, both $\beta_{c}$ and $\beta_{c}^{A}$ increase exponentially with the increase of $h$. Furthermore, the negative correlation between the average individual activity $\langle a\rangle$ and the outbreak threshold $\beta_{c}^{A}$ can be demonstrated in Figures 5(c) and 5(d).

\section{Conclusions}

In our paper, we study rumor spreading process with the social reinforcement mechanism on both random networks and 
activity-driven networks. The effects of the reinforcement mechanism on the infection prevalence and the outbreak threshold of rumor spreading are investigated. Two spreading models with both internal and external reinforcement factors are established on both random networks and $\mathrm{AD}$ networks. The internal reinforcement mechanism is only dependent on the self-enhancement rate. For the external reinforcement mechanism, the enhancement effect is related to the infection rate and it varies with the dynamic infection level in the network. We analyze the outbreak threshold of rumor spreading process on both analytical and numerical methods. Results show that both the infection rate and the self-enhancement rate are positively correlated with the outbreak scale and negatively correlated with the outbreak threshold. In addition, we have investigated the impacts of network topology on rumor spreading under the framework of both the random network and the AD network. Our study demonstrates that the existence of intermediate states in the reinforcement process will hinder the rumor spreading. In the future works, the heterogeneous number of intermediate state for different individuals will be further explored.

\section{Data Availability}

All data can be accessed in the numerical simulation section of this article.

\section{Conflicts of Interest}

The authors declare that they have no conflicts of interest.

\section{Acknowledgments}

This research was funded by National Natural Science Foundation of China (No. 61801518 and No. 61873194) and Natural Science Foundation of Hubei Province (No. 2017CFB661).

\section{References}

[1] B. Pittel, "On spreading rumor," SIAM Journal on Applied Mathematics, vol. 47, no. 1, pp. 213-223, 1987.

[2] M. A. Amaral and J. J. Arenzon, "Rumor propagation meets skepticism: A parallel with zombies," Europhysics Letters, vol. 124, no. 1, 18007 pages, 2018.

[3] L. Huo and Y. Cheng, "The impact of media coverage and emergency strategies on the rumor spreading," Discrete Dynamics in Nature and Society, vol. 2018, Article ID 4137129, 14 pages, 2018.

[4] J. M. Tchuenche, N. Dube, C. P. Bhunu, R. J. Smith, and C. T. Bauch, "The impact of media coverage on the transmission dynamics of human influenza," BMC Public Health, vol. 11, no. Suppl 1:S5, 2011.

[5] F. Chierichetti, S. Lattanzi, and A. Panconesi, "Rumor spreading in social networks," in Proceedings of the International Colloquium on Automata, Languages, and Programming ICALP, Springer, Greece, 2009.
[6] B. Doer, M. Fouz, and T. Friedrich, "Why rumors spread so quickly in social networks," Communications of the ACM, vol. 55, no. 6, 70 pages, 2012.

[7] X.-X. Zhao and J.-Z. Wang, "Dynamical behaviors of rumor spreading model with control measures," Abstract and Applied Analysis, vol. 2014, Article ID 247359, pp. 1-11, 2014.

[8] S. Dong and Y.-C. Huang, "A class of rumor spreading models with population dynamics," Communications in Theoretical Physics, vol. 70, no. 6, p. 795, 2018.

[9] D. Trpevski, W. K. S. Tang, and L. Kocarev, "Model for rumor spreading over networks," Phys Rev E, vol. 81, no. 5 Pt 2, p. 056102 , May 2010.

[10] L. Zhao, H. Cui, X. Qiu, X. Wang, and J. Wang, "SIR rumor spreading model in the new media age," Physica A: Statistical Mechanics and its Applications, vol. 392, no. 4, pp. 995-1003, 2013.

[11] B. Il Hong, N. Hahm, and S.-H. Choi, "SIR Rumor spreading model with trust rate distribution," Networks \& Heterogeneous Media, vol. 13, no. 3, pp. 515-530, 2018.

[12] Z. Zhang, Y. Wang, and L. Guerrini, "Bifurcation analysis of a delayed worm propagation model with saturated incidence," Advances in Mathematical Physics, vol. 2018, Article ID 7619074, 9 pages, 2018.

[13] C. Lazureanu and C. Petrisor, "Stability and energy-casimir mapping for integrable deformations of the kermackmckendrick system," Advances in Mathematical Physics, vol. 2018, Article ID 5398768, 9 pages, 2018.

[14] L. Zhao, J. Wang, Y. Chen, Q. Wang, J. Cheng, and H. Cui, "SIHR rumor spreading model in social networks," Physica A: Statistical Mechanics and its Applications, vol. 391, no. 7, pp. 2444-2453, 2012.

[15] Y. Hu, Q. Pan, W. Hou, and M. He, "Rumor spreading model with the different attitudes towards rumors," Physica A: Statistical Mechanics and its Applications, vol. 502, pp. 331-344, 2018.

[16] D. Li and J. Ma, "How the governments punishment and individuals sensitivity affect the rumor spreading in online social networks," Physica A: Statistical Mechanics and its Applications, vol. 469, pp. 284-292, 2017.

[17] H. Wang, L. Deng, F. Xie, H. Xu, and J. Han, "A new rumor propagation model on SNS structure," in IEEE International Conference on Granular Computing, pp. 499-503, Hangzhou, China, 2012.

[18] J. Ma, D. Li, and Z. Tian, "Rumor spreading in online social networks by considering the bipolar social reinforcement," Physica A: Statistical Mechanics and its Applications, vol. 447, pp. 108-115, 2016.

[19] J. Zhou, Z. Liu, and B. Li, "Influence of network structure on rumor propagation," Physics Letters A, vol. 368, no. 6, pp. 458-463, 2007.

[20] M. Nadini, A. Rizzo, and M. Porfiri, "Epidemic spreading in temporal and adaptive networks with static backbone," IEEE Transactions on Network Science and Engineering, pp. 1-1, 2018.

[21] T. Hiraoka and H.-H. Jo, "Correlated bursts in temporal networks slow down spreading," Scientific Reports, vol. 8, no. 1, Article ID 15321, 2018.

[22] N. Perra, B. Gonçalves, R. Pastor-Satorras, and A. Vespignani, "Activity driven modeling of time varying networks," Scientific Reports, vol. 2, no. 1, 2012.

[23] P. Hu, L. Ding, and X. An, "Epidemic spreading with awareness diffusion on activity-driven networks," Physical Review E, vol. 98 , no. $6,2018$. 
[24] H. Zhu, J. Ma, and S. Li, "Effects of online and offline interaction on rumor propagation in activity-driven networks," Physica A: Statistical Mechanics and its Applications, vol. 525, pp. 11241135, 2019.

[25] P. van den Driessche and J. Watmough, "Reproduction numbers and sub-threshold endemic equilibria for compartmental models of disease transmission," Mathematical Biosciences, vol. 180, no. 1-2, pp. 29-48, 2002. 


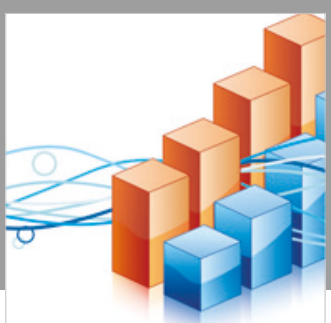

Advances in

Operations Research

\section{-n-m}
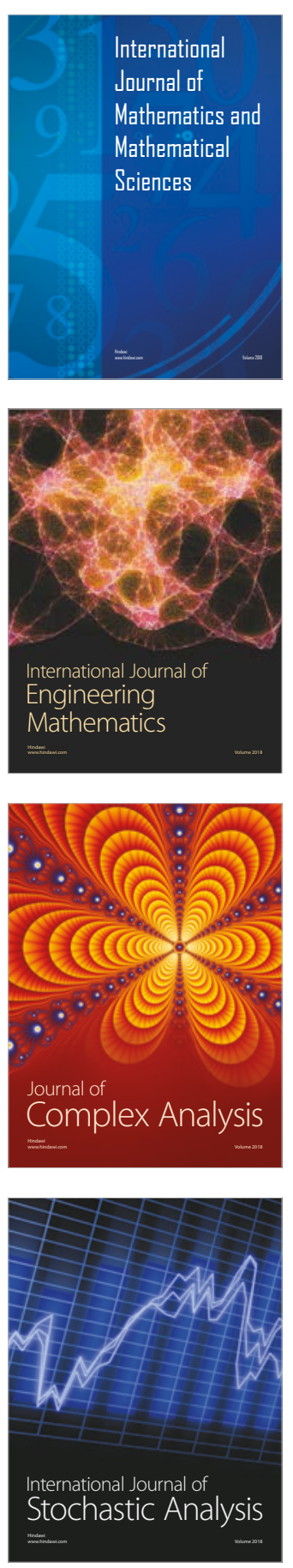
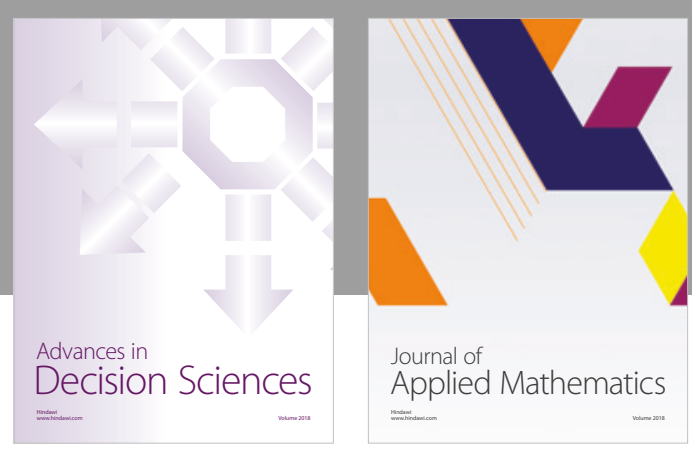

Journal of

Applied Mathematics
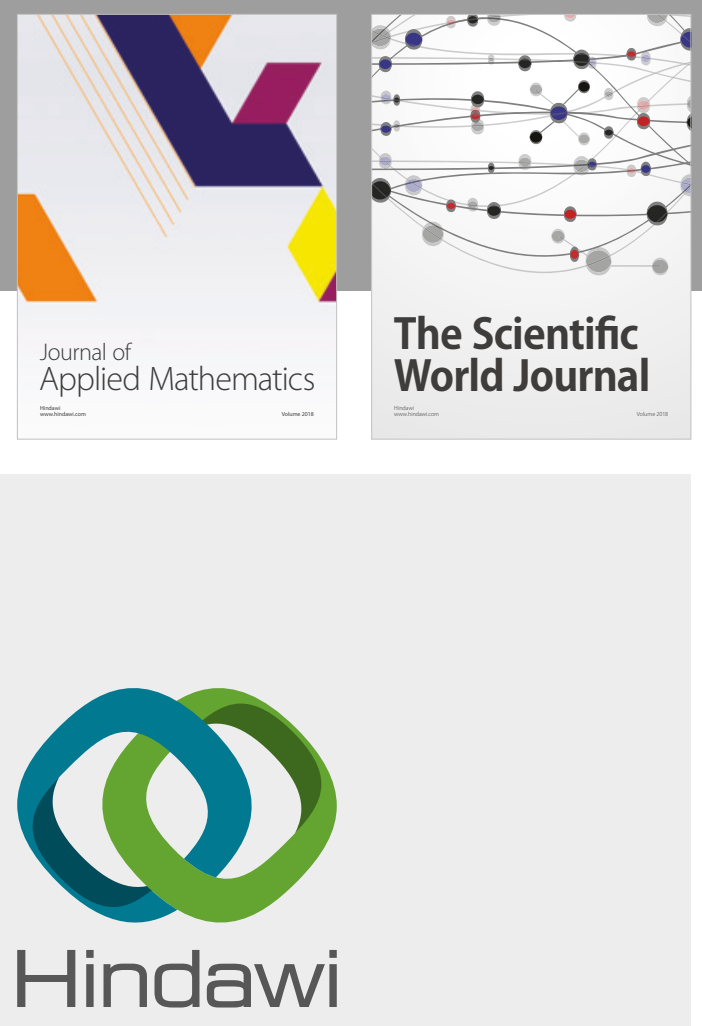

Submit your manuscripts at

www.hindawi.com

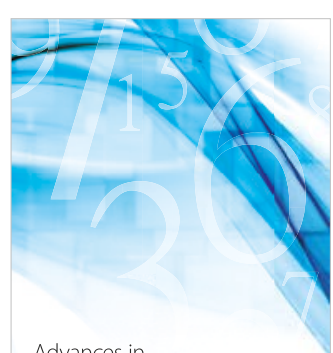

Advances in
Numerical Analysis
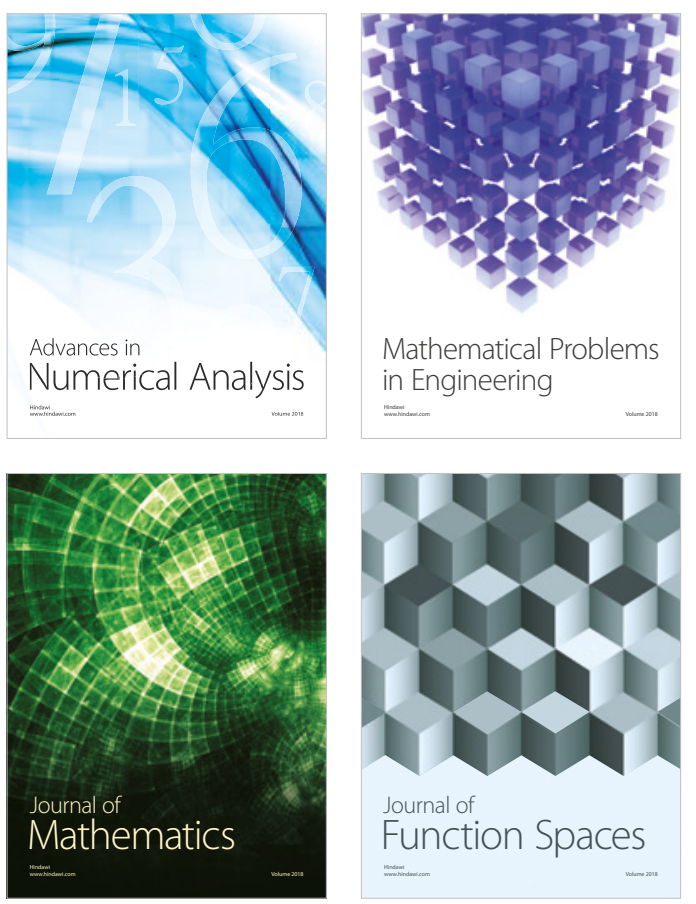

Mathematical Problems in Engineering

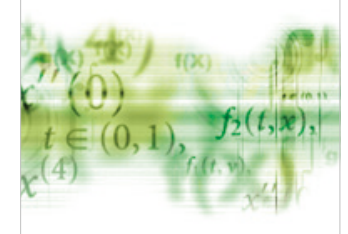

International Journal of

Differential Equations

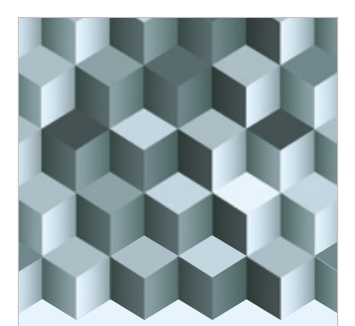

Journal of

Function Spaces
The Scientific

World Journal

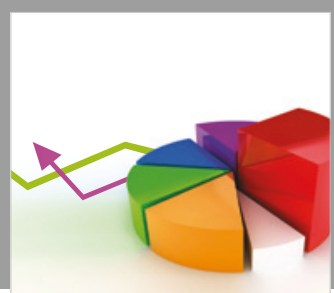

Journal of

Probability and Statistics
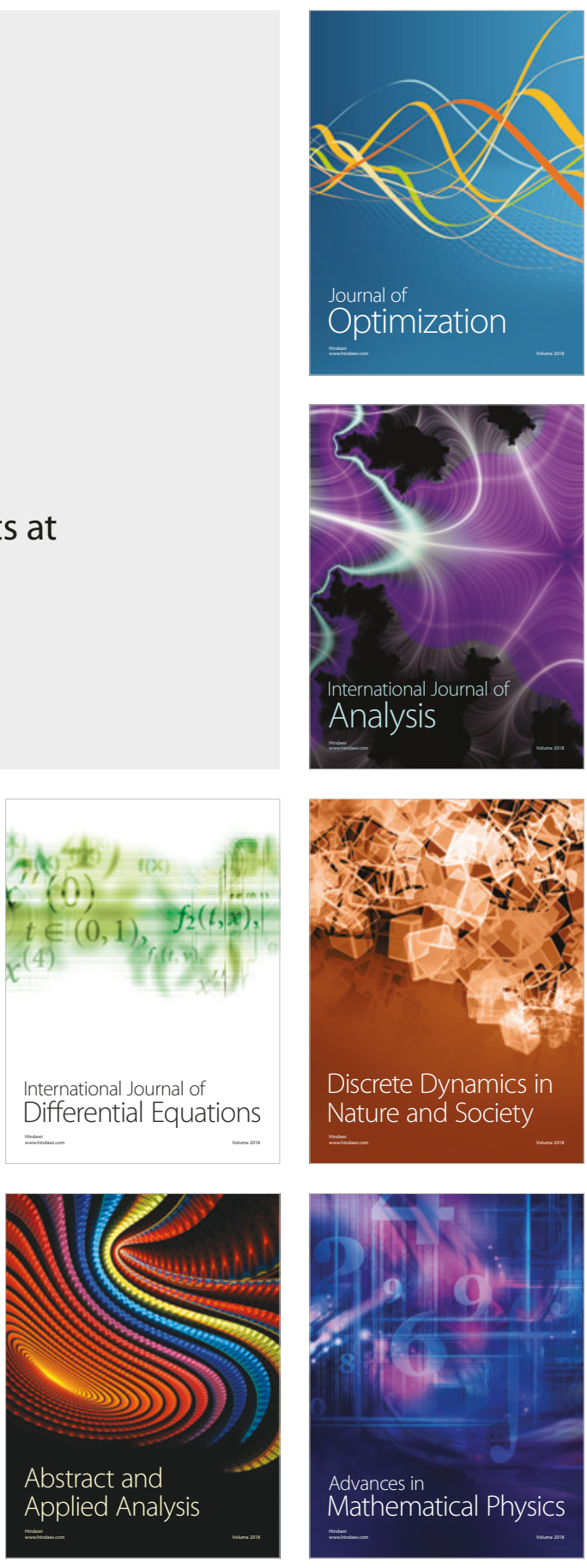